\title{
Gravitational wave emission from oscillating millisecond pulsars
}

\author{
Mark G. Alford and Kai Schwenzer \\ Department of Physics, Washington University, St. Louis, Missouri, 63130, USA
}

\begin{abstract}
Neutron stars undergoing r-mode oscillation emit gravitational radiation that might be detected on earth. For known millisecond pulsars the observed spindown rate imposes an upper limit on the possible gravitational wave signal of these sources. Taking into account the physics of r-mode evolution, we show that only sources spinning at frequencies above a few hundred Hertz can be unstable to r-modes, and we derive a more stringent universal r-mode spindown limit on their gravitational wave signal, exploiting the fact that the r-mode saturation amplitude is insensitive to the structural properties of individual sources. We find that this refined bound limits the gravitational wave strain from millisecond pulsars to values below the detection sensitivity of next-generation detectors. Young sources are therefore a more promising option for the detection of gravitational waves emitted by r-modes and to probe the interior composition of compact stars in the near future.
\end{abstract}

\section{INTRODUCTION}

With the significant sensitivity improvement of forthcoming next generation gravitational wave detectors like advanced LIGO [1], advanced Virgo [2] and the LCGT [3] there is a realistic chance that gravitational waves may be directly observed. In addition to transient events such as neutron star and/or black hole mergers or supernovae, which require that an event happens sufficiently near to us during the observation period [4], it is important to also consider continuous sources. Millisecond pulsars are a particularly promising class since they are very old and stable systems and therefore could be reliable sources of gravitational waves. Their fast rotation strongly favors gravitational wave emission [5], and the fact that their timing behavior is known to high precision [6] greatly simplifies the analysis required to find a signal in the detector data. Emission due to deformation of these objects ("mountains"), which is usually parametrized by an ellipticity, is the standard paradigm for continuous gravitational wave searches [7, but global oscillation modes of a star can also emit copious gravitational waves that could be detectable if the oscillation reaches sufficiently high amplitudes. R-modes are the most interesting class [8-11, because they are generically unstable in millisecond pulsars and therefore will be present unless the dissipative damping is strong enough.

If r-modes arise in a spinning neutron star, they affect the spindown (since they cause the star to lose angular momentum via gravitational radiation) and the cooling (since the the damping forces on the r-mode generate heat). To understand the interplay of these effects we have developed [12, 13] an effective description of the spindown evolution where complicated details about the star's interior are absorbed into a few effective parameters. The resulting spindown can be rather different from that predicted by simpler approaches, and includes strict bounds on the uncertainties in the final results. In this paper we will use this method to analyze the possible $r-$ mode gravitational radiation of old neutron stars. Firstly, however, we provide some background and motivation.

R-modes can occur in young or old pulsars. In the case of young sources [5, 14 -16] we have analyzed their rmode evolution 12 and found that $\mathrm{r}$-modes can provide a quantitative explanation for their observed low spin rates. Moreover, the r-mode gravitational emission is expected to be strong, because a large r-mode amplitude would be required to spin down the known young pulsars to their current low spin frequencies within their lifetimes which are as short as a thousand years. These known pulsars are no longer in their r-mode spindown epoch, but there may be unobserved young neutron stars, e.g. associated with known supernova remnants such as SN 1987A, that are currently undergoing r-mode spindown, and several of them would be in the sensitivity range of advanced LIGO [12], allowing this scenario to be falsified by future measurements.

In this paper we focus on old neutron stars which have been spun up by accretion, and we perform an analysis of their expected r-mode gravitational wave radiation. In 13] novel r-mode instability regions in spindown timing parameter space have been derived that allow us to decide if r-modes can be present in old millisecond radio pulsars. As discussed there, there are two scenarios to explain the observed timing data. It might be that the ordinary nuclear matter model of neutron stars is incomplete, and there is additional damping (e.g. from exotic forms of matter or currently overlooked physical processes) that stops r-modes from growing in these stars. In this case there will be no r-mode gravitational radiation from old neutron stars. The other possibility is the conventional scenario where only standard damping mechanisms are present in neutron stars. In this scenario most old millisecond pulsars will be undergoing r-mode oscillations, since for expected r-mode saturation amplitudes the dissipative heating ensures that fast spinning sources can neither cool nor spin out of the parameter region were r-modes are unstable [13. Yet, some slower spinning sources can escape the instability region and we will determine the limiting frequency. Therefore, there will be gravitational radiation from most old neutron stars in this scenario, and the purpose of this paper is to find out whether it could be detected on earth.

The detectability of known continuous sources is generally described by the "spindown limit" which is, for a 
specific source with known timing data, the maximum gravitational wave strain that can be emitted by that source. Despite the quite restrictive limits set by the spindown data, the large spin frequencies of millisecond pulsars could nevertheless lead to a detectable signal. Present gravitational wave detectors-like the original LIGO interferometer - did not probe the spindown limit for millisecond pulsars. However, next generation detectors including the advanced LIGO detector will be able to beat the spindown limit for various sources. Therefore, it is interesting to assess the chance to detect gravitational emission from oscillating millisecond pulsars.

We will introduce here the universal r-mode spindown limit on the gravitational wave strain, which is more restrictive since it takes into account our understanding of the r-mode spindown and the complete information we have about these systems. Whereas deformations of a given source depend on its evolutionary history and could therefore vary significantly from one source to another, for proposed saturation mechanisms the r-mode saturation amplitude proves to be rather insensitive to details of a particular source, like its mass or radius [1719]. The expected gravitational wave strain of a given source can then be strongly constrained by the timing data of the entire set of millisecond pulsars. Using our semi-analytic approach to pulsar evolution, and assuming that the same saturation and cooling mechanism (with given power-law dependence on temperature) operates in all the stars, we can then obtain the universal limit given in Eq. 12. We will see that this is considerably below the standard spindown limits, indicating that it will be harder than previously expected to see r-mode gravitational waves from these sources.

\section{R-MODE SPINDOWN OF MILLISECOND PULSARS}

As described in [12, 13] the r-mode evolution [11] can be discussed within an effective description, which relies on the fact that a compact star appears effectively as a point source and that the relevant material properties integrated over the star have simple power law dependencies on the macroscopic observables that change during the evolution. The relevant macroscopic quantities are the power emitted as gravitational waves $P_{G}$, the dissipated power $P_{D}$ that heats the star and the thermal luminosity $L$ that cools the star

$$
P_{G}=\hat{G} \Omega^{8} \alpha^{2}, P_{D}=\hat{D} T^{\delta} \Omega^{\psi} \alpha^{\phi}, L=\hat{L} T^{\theta},
$$

in terms of the rotational angular velocity $\Omega=2 \pi f$, the core temperature $T$ of the star and the dimensionless rmode amplitude $\alpha$ defined in [10, 12]. The explicit form of the prefactors $\hat{G}, \hat{D}$ and $\hat{L}$ for different damping and cooling mechanisms 13 is given in tab. I]

R-modes are unstable and their fast growth has to be stopped by a non-linear dissipative saturation mechanism. Even though there are several interesting proposals

\begin{tabular}{|c||c|}
\hline parameter of the ... & integral expression \\
\hline \hline GW luminosity & $\hat{G} \equiv \frac{2^{17} \pi}{3^{8} 5^{2}} \tilde{J}^{2} G M^{2} R^{6}$ \\
\hline \hline Shear visc. dissipation & $\hat{D}=5 \tilde{S} \Lambda_{\mathrm{QCD}}^{3+\sigma} R^{3}$ \\
\hline Bulk visc. dissipation & $\hat{D}=\frac{2^{3}}{3^{3} 7} \frac{\Lambda_{\mathrm{QCD}}^{9-\delta} \tilde{V} R^{7}}{\Lambda_{\mathrm{EW}}^{4}}$ \\
\hline Ekman layer dissipation & $\hat{D}=5\left(\frac{2}{3}\right)^{\frac{9}{2}} \frac{3401+2176 \sqrt{2}}{11 ! !} \sqrt{\hat{\eta}_{c} \rho_{c}} R_{c}^{4}$ \\
\hline \hline Neutrino luminosity & $\hat{L}=\frac{4 \pi R^{3} \Lambda_{\mathrm{QCD}}^{9-\theta} \tilde{L}}{\Lambda_{\mathrm{EW}}^{4}}$ \\
\hline Photon luminosity & $\hat{L}=\frac{\pi^{3}}{15} R^{2} \hat{X}^{4}$ \\
\hline
\end{tabular}

Table I: Parameters in the general parameterization eq. (1) for the energy loss rates. The arising quantities are the mass $M$ and the radius $R$ of the star, the gravitational constant $G$, generic normalization scales $\Lambda_{\mathrm{QCD}}$ and $\Lambda_{\mathrm{EW}}$ and in case of Ekman damping the relevant quantities at the crust/core interface, see 20]. The dimensionless constants $\tilde{J}, \tilde{V}, \tilde{S}$ and $\tilde{L}$ contain the complete information about the interior of the star. Their definition and values for realistic neutron stars are given in 12 .

[17 19, 21 24 it is not yet settled which mechanism will dominate and saturate r-modes. For millisecond pulsars we expect moderate saturation amplitudes, in which case the pulsar spindown is determined by the equation 11.

$$
\frac{d \Omega}{d t}=-\frac{3 \hat{G} \alpha_{\mathrm{sat}}^{2}(T, \Omega)}{I} \Omega^{7}-\cdots
$$

in terms of the moment of inertia of the star $I$ and the r-mode saturation amplitude $\alpha_{\text {sat }}$. The observed total spindown rate will in general be larger since in addition to r-modes there are other spindown mechanisms given by the ellipsis. Nevertheless, by assuming that the observed spindown rate is entirely due to r-modes, observed pulsar timing data allows one to give upper bounds on the r-mode saturation amplitude. These bounds are shown for the observed radio pulsars included in the ATNF database [6] in fig. 1] and they require very low saturation amplitudes, $10^{-7} \lesssim \alpha_{\text {sat }} \lesssim 10^{-5}[13$, 17. Similar low values are obtained from pulsars in low mass $\mathrm{x}$-ray binaries [13, 25]. Moreover, one can see in fig. 1 that faster spinning sources generally set more stringent bounds on the saturation amplitude of r-modes in the considered pulsar.

The r-mode saturation amplitude can in general depend both on the temperature and the frequency of the star. We use a general parametrization of the saturation amplitude with a power-law form

$$
\alpha_{\text {sat }}(T, \Omega)=\hat{\alpha}_{\text {sat }} T^{\beta} \Omega^{\gamma},
$$

as realized for the proposed saturation mechanisms [1719, 23. Here the exponents are fixed (rational) numbers determined by the saturation mechanism, whereas the reduced amplitude $\hat{\alpha}_{\text {sat }}$ is less well known and can also depend on parameters of the particular source, like the mass or the radius. Using this general approach it was found in 12 that the r-mode heating is significant even for small amplitude modes and the thermal evolution is 


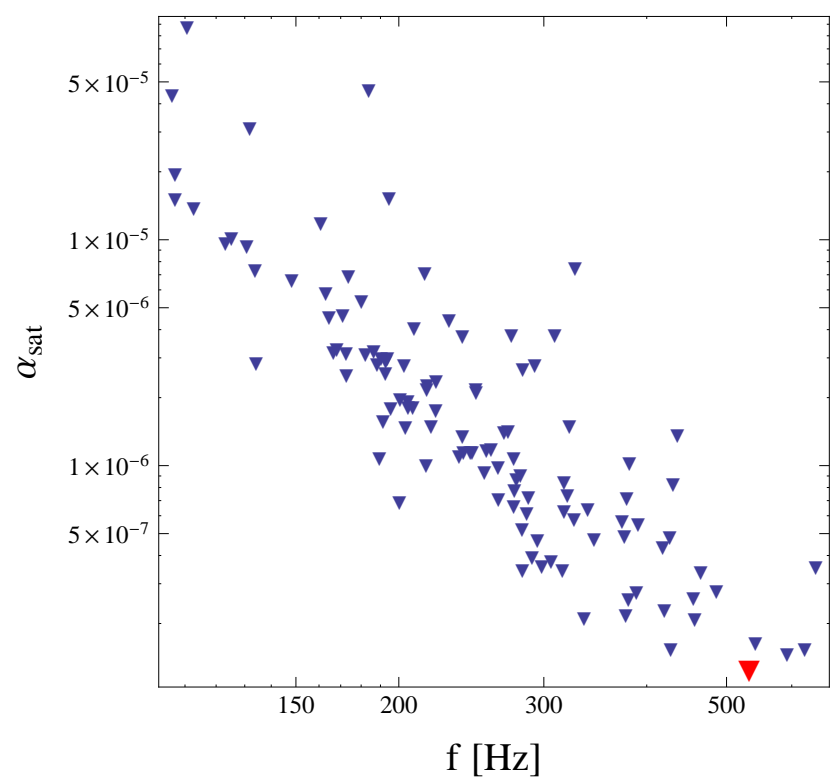

Figure 1: Upper bounds on the r-mode saturation amplitude arising from the observed spindown of the pulsars in the ATNF database [6]. The strongest bound $\alpha_{\text {sat }} \lesssim 1.2 \times 10^{-7}$ is obtained for the $533 \mathrm{~Hz}$ pulsar J0034-0534 which has a spindown rate $\dot{f} \approx-1.4 \cdot 10^{-15} s^{-2}$ (large triangle at lower right, red online).

systematically faster than the spindown. Therefore the star reaches a thermal steady state where the dissipative r-mode heating balances the cooling due to photons and neutrinos and the temperature is given by

$$
T_{\mathrm{hc}}=\left(\frac{\hat{G} \hat{\alpha}_{\mathrm{sat}}^{2} \Omega^{8+2 \gamma}}{\hat{L}}\right)^{\frac{1}{\theta-2 \beta}} .
$$

This leads to a spindown equation along this steady state curve [12, 13 .

$$
\frac{d \Omega}{d t}=-\frac{3 \hat{G}^{\theta /(\theta-2 \beta)} \hat{\alpha}_{\mathrm{sat}}^{2 \theta /(\theta-2 \beta)}}{I \hat{L}^{2 \beta /(\theta-2 \beta)}} \Omega^{n_{r m}},
$$

with an effective braking index

$$
n_{\mathrm{rm}}=7\left(\frac{1+2 \gamma / 7+2 \beta /(7 \theta)}{1-2 \beta / \theta}\right)
$$

that depends on the saturation mechanism and can be rather different from the generic r-mode spindown exponent 7 .

The spindown equation has the solution

$$
\begin{aligned}
\Omega(t) & =\left(\left(\Omega_{i}\right)^{z}+\frac{3 z}{2}\left(\frac{\hat{G}^{\theta} \hat{\alpha}_{\mathrm{sat}}^{2 \theta}}{\hat{L}^{2 \beta}}\right)^{\frac{1}{\theta-2 \beta}} \frac{t-t_{i}}{I}\right)^{1 / z} \\
z & \equiv \frac{2(3+\gamma) \theta+4 \beta}{\theta-2 \beta}
\end{aligned}
$$

This solution has two limits. At early times the first term in the parenthesis of eq. (7) dominates, so that the star hardly spins down, i.e. $\Omega \approx \Omega_{i}$, and at late times the second term dominates, so that the spindown becomes independent of the initial angular velocity $\Omega_{i}$. The crossover point between these two regimes is determined by the reduced saturation amplitude $\hat{\alpha}_{\text {sat }}$. For young pulsars the $\Omega_{i}$-independent late time behavior of the spindown law is relevant. For some old millisecond pulsars the spindown rate is so low that the "early time" regime is realized and they hardly spin down even over their billion year age.

The r-mode evolution eq. 77) takes place unless the dissipation, which depends strongly on temperature and frequency, is strong enough to completely damp r-modes. R-modes are only unstable at sufficiently high frequencies: a typical instability region for a neutron star with standard damping mechanisms in a $T-\Omega$-diagram [10] is shown in fig. 2. By "standard damping" we mean established mechanisms ${ }^{1}$, namely shear viscosity due to leptonic and hadronic scattering [26] and bulk viscosity due to modified Urca reactions [27]. Ref. 28] gives a general semi-analytic expression for the minimum frequency $\Omega_{\text {min }}$ down to which r-modes can be unstable, and shows that this limit is extremely insensitive to unknown details of the source and the microphysics (see also [10]). Fig. 2 also shows two qualitatively different evolution trajectories. A recycled millisecond pulsar entering the instability region at point $\mathrm{A}$ in fig. 2 is slowly spun up and kept warm by accretion in a binary system, following the thick vertical line. The r-mode evolution, eq. (7), starts when accretion stops. This may occur when the star is spinning slowly (B) or quickly (C). In either case, even though the star is in the region of $\Omega-T$ space where according to the standard damping mechanism r-modes are unstable, the star then cools faster than it spins down (following the thin horizontal lines). If accretion brought the star to a high spin frequency (C) then the star cools until it reaches the steady state line (dashed line, given by eq. (4) at point (D); it then slowly spins down, following the steady-state line, and would only reach the boundary of the instability (E) after time scales that are longer than the age of known sources. Therefore we expect such a source not too far below point (D). Fig. 2 shows a steady-state line for low r-mode saturation amplitude, in which case the line is high enough that it exits

\footnotetext{
1 A potential Ekman layer at the crust-core boundary 20] does not qualitatively change this picture 13 .
} 
the instability region at a frequency $\Omega_{f}$ which is significantly above the minimum frequency $\Omega_{\min }$. This means that if accretion leaves the star with a low spin frequency (B), below $\Omega_{f}$, then the star cools in less than a million years [29] and reaches the boundary of the instability region $(\mathrm{F})$. The value of $\Omega_{f}$ is

$$
\Omega_{f}=\left(\frac{\hat{D}^{\theta-2 \beta} \hat{\alpha}_{\mathrm{sat}}^{2 \delta}}{\hat{G}^{\theta-\delta-2 \beta} \hat{L}^{\delta}}\right)^{\frac{1}{6 \theta-8 \delta-12 \beta-2 \delta \gamma}}
$$

It was shown in 12, 13, that this expression is extremely insensitive to the microphysical details. Whereas for young sources discussed in 12 neutrino emission is the relevant cooling mechanism to determine the final spindown frequency, for the low saturation amplitudes relevant for millisecond pulsars photon cooling from the surface and damping due to shear viscosity dominates [13] in eq. (8). For a given upper bound on $\hat{\alpha}_{\text {sat }}$, all sources below this universal $r$-mode frequency bound $\Omega_{f}$ cannot be undergoing r-mode spindown since they either have been spun out of the instability region or cooled out of it in less than a million years, which is considerably shorter than their billion year age. In contrast, all sources spinning faster than $\Omega_{f}$ must be undergoing $\mathrm{r}-$ mode spindown (i.e. they are on the steady-state curve in fig. 2). The fastest spinning sources $(f \gtrsim 600 \mathrm{~Hz})$ could have only left the instability region if the saturation amplitude would be as low as $\alpha_{\text {sat }} \lesssim 10^{-10}[13$, which is orders of magnitude below what proposed saturation mechanisms can provide [17, 19, 23]. We conclude that fast spinning sources should be emitting gravitational waves via r-mode spindown and we will determine the required spin frequencies below.

\section{GRAVITATIONAL WAVE STRAIN}

R-modes emit gravitational waves due to their timevarying current quadrupole moment. The gravitational wave frequency $\nu$ emitted by the dominant fundamental $(m=2)$ r-mode is related to the rotational angular velocity via $\nu=2 /(3 \pi) \Omega[11$. The gravitational wave signal of a given source is described by the intrinsic gravitational wave strain of the detector, which describes the expected signal in a terrestrial detector and can directly be compared to the detector noise. For r-modes it takes the form 30

$$
h_{0}=\sqrt{\frac{2^{15} \pi^{7}}{5}} \frac{\tilde{J} G M R^{3} \nu^{3} \alpha_{\mathrm{sat}}}{D},
$$

where $D$ is the distance to the source. In a recent study of the gravitational wave emission of young sources [12] it was found that for the large amplitudes required to explain the low spin frequencies of young pulsars, the late time behavior of the spindown evolution eq. (7) is relevant and in this case the strain eq. (9) depends only on

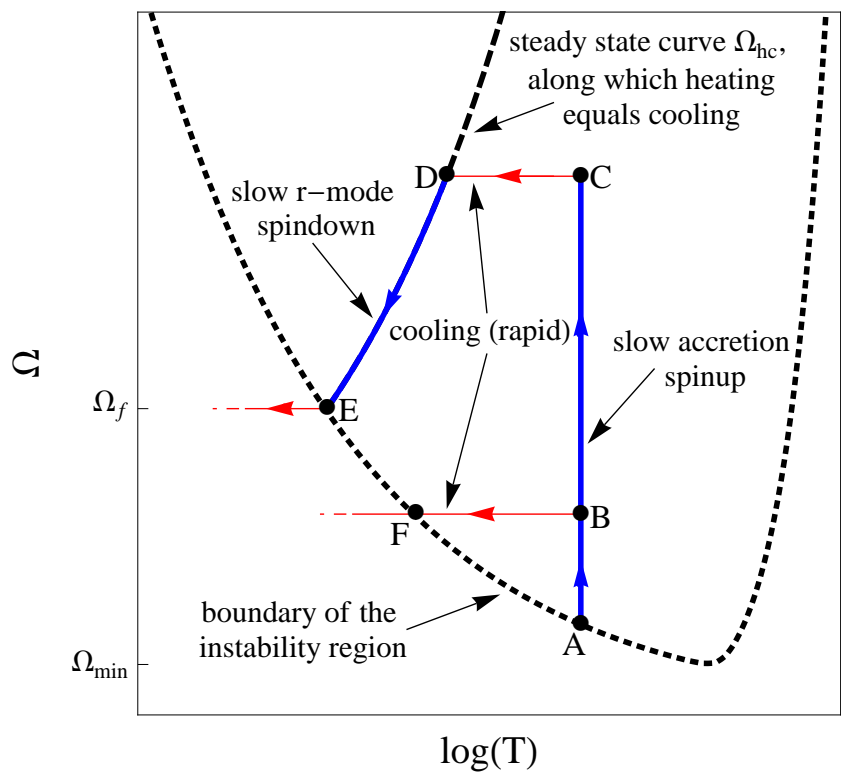

Figure 2: Schematic evolution of recycled radio pulsars which have been spun up by accretion. Whereas the cooling (horizontal segments) takes less than a million years, the slow spindown along the steady state curve takes longer than a billion years. The occurrence of a long r-mode spindown epoch is determined by whether the frequency when accretion ends is below (B) or above (C) the universal r-mode frequency bound $\Omega_{f}$. See the text for details.

the age and the distance of the source [16], and is independent of the saturation amplitude. Because of the restrictive bounds on the saturation amplitude shown in fig. 1] for some old radio pulsars the "early time" limit of the evolution is relevant, where the frequency barely changes and the strain depends linearly on the saturation amplitude. In fig. 2 such a source stays close to its starting point $\mathrm{D}$ on the spindown curve for more than a billion years. However, in general the time evolution is relevant and has to be taken into account. This can be seen in fig. 3 where the evolution of the gravitational wave strain is shown for saturation amplitudes relevant for millisecond pulsars. The dots also show for various amplitudes the end of the gravitational wave emission, where the source spins slowly enough that the r-mode is damped. As seen, for $\alpha_{\text {sat }}<10^{-5}$ the time needed for a star to spin out of the r-mode instability region is considerably more than a billion years, longer than the age of these sources.

\section{A. Standard r-mode spindown limit}

Using the spindown equation (2) to eliminate the rmode saturation amplitude $\alpha_{\text {sat }}$ in eq. (9), i.e. employing 


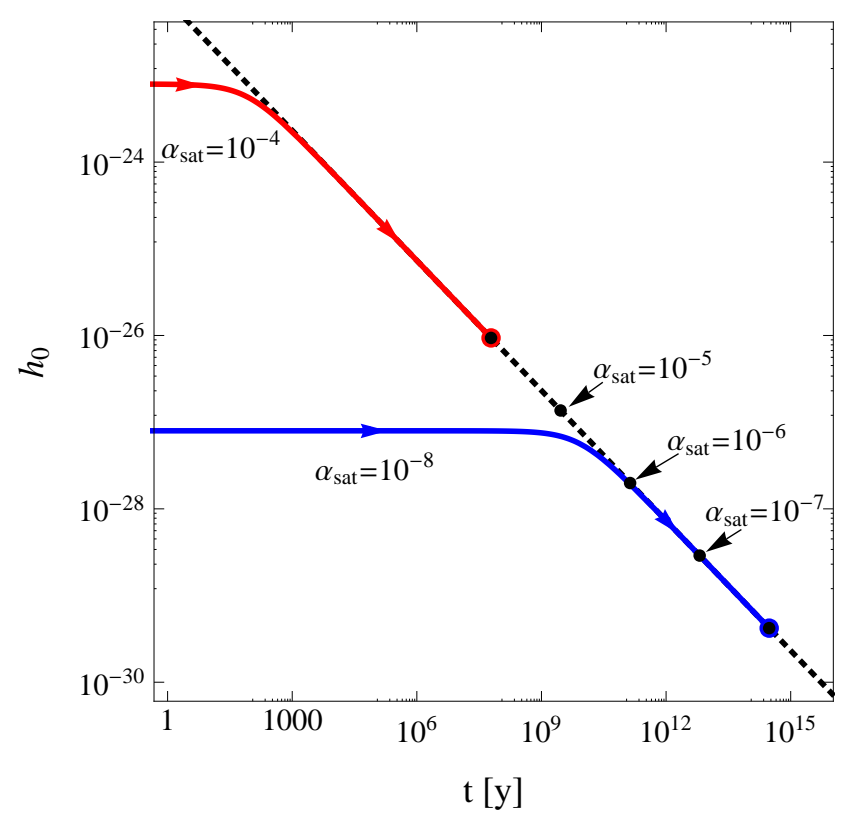

Figure 3: The time evolution of the emitted intrinsic gravitational wave strain amplitude (solid curves) and the endpoints of the gravitational wave emission (dots) [12] shown for different saturation amplitudes and for a fiducial source located at a distance of $1 \mathrm{kpc}$. The universal late time behavior is also shown (dotted line).

the values given in fig.11, yields the spindown limit $^{2}$ [5, 30]

$$
h_{0}^{(\mathrm{sl})}=\sqrt{\frac{15}{4} \frac{G I|\dot{\Omega}|}{D^{2} \Omega}},
$$

which provides an upper bound that is saturated when the entire rotational energy loss is due to the gravitational wave emission and accompanying dissipation caused by r-modes. The spindown limits for the observed radio pulsar data [6] are shown in fig. 4 and are represented by inverted triangles. Here the spindown limits from r-modes (solid triangles) are compared to those for the typically considered case of elliptic star deformations (open triangles), which has recently been studied in detail by the LIGO collaboration [5]. R-modes lead to a slightly higher strain but at a lower frequency [30]. These limits are also compared to the detector sensitivity at $95 \%$ confidence limit (curves), given by $h_{0}^{95 \%} \approx 10.8 \sqrt{S_{h} / \Delta t}[5$ ] in terms of the spectral density $S_{h}$ of the detector strain noise and the observation interval $\Delta t$. To assess fig. 4 it is

\footnotetext{
2 The expression given here is slightly smaller than the estimate given in [30, since the rotational energy loss goes not entirely into gravitational waves but is partly dissipated to saturate the r-mode at a finite amplitude.
}

important to recall that $r$-modes are only unstable at sufficiently large frequencies. The lowest frequency at which r-modes are unstable $\left(\Omega_{\min }\right.$ in Fig. 2) [10, 28] shown by the vertical line, sets a strict frequency limit below which no r-mode gravitational wave emission is possible.

Despite this restriction, the figure shows that the spindown limit for several millisecond radio pulsars should be beaten by the advanced LIGO detector. However, even though the pulsars J0537-6910 and J0437-4715 could be significantly above the detector sensitivity they are not promising sources. The pulsar J0537-6910 is actually a young pulsar that has been analyzed in detail in [12] where it is shown that although it is slightly above the minimum frequency of the instability region, it is very likely outside of it. The $f=174 \mathrm{~Hz}$ pulsar J0437-4715 is the closest and brightest millisecond pulsar and therefore would be a natural target. However, this is also the only non-accreting source for which a temperature estimate is available [31, and this shows that it is outside of the instability region and similarly cannot emit gravitational waves due to r-modes. Moreover, its frequency is below likely values of $\Omega_{f}$ (eq. (8p) so, as we discussed at the end of section [II and will analyze in more detail below, it ought to have already cooled out of the instability region for a neutron star with standard damping mechanisms [13. Thinking beyond the advanced LIGO sensitivity thresholds shown in fig. 4 , we note that planned detectors like the Einstein telescope, which has an order of magnitude higher sensitivity, would be able to detect the gravitational waves that would be emitted from many sources, if r-modes are responsible for the better part of their observed spindown rate.

\section{B. Universal r-mode spindown limit}

The spindown limit for a particular source only takes into account information about that source. Here we will derive a more restrictive limit taking into account the entire data set of radio pulsars. It is based on the observation that proposed r-mode saturation mechanisms are very insensitive to the details of a particular source [17-19]. To make this statement quantitative, we factorize the reduced saturation amplitude given in eq. (3) by writing

$$
\hat{\alpha}_{\mathrm{sat}}=\hat{\alpha}_{\mathrm{sat}}^{(\mathrm{mic})} \hat{\alpha}_{\mathrm{sat}}^{(\mathrm{mac})}
$$

where $\hat{\alpha}_{\mathrm{sat}}^{(\mathrm{mic})}$ depends on the microphysics of the saturation mechanism and is source-independent, and $\alpha_{\text {sat }}^{(\mathrm{mac})}$ depends on the macroscopic properties of a specific source (mass, radius, etc) ${ }^{3}$, which generically only vary within

\footnotetext{
3 To make the factorization unambiguous, we will use the convention that once a set of macroscopic parameters have been chosen, the source-dependent macroscopic part $\alpha_{\text {sat }}^{(\mathrm{mac})}$ consists of pow-
} 


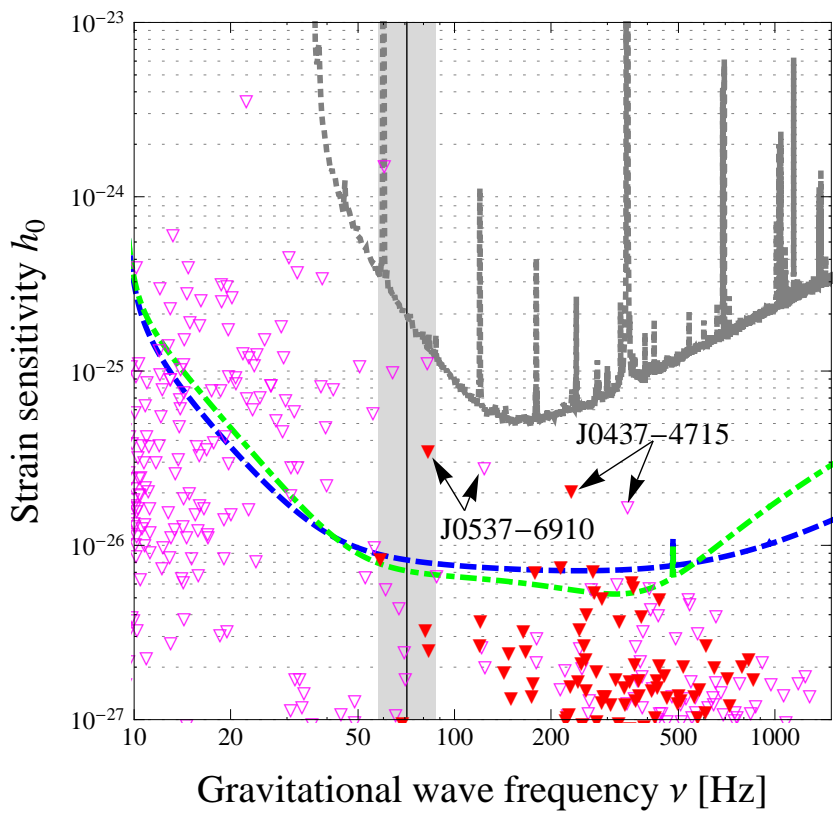

Figure 4: The standard spindown limits of known radio pulsars compared to the characteristic strain amplitude for different detector configurations assuming a coherent analysis of a year of data. Open (magenta) triangles show the limits for the standard case of elliptic deformations of the star [5] and filled (red) triangles for the case of r-mode gravitational wave emission. The solid (grey) curve gives the sensitivity of the original LIGO detector and the dashed (blue) and dot-dashed (green) curves show the sensitivity of the advanced LIGO detector in the standard and neutron star enhanced mode. The vertical line shows the limiting frequency below which r-modes are absent in a neutron star and the shaded band gives the uncertainty on it using the semi-analytic result 28 and the ranges for the underlying parameters used in [12].

narrow margins. For a given saturation mechanism determined by $\beta$ and $\gamma$ in eq. (3) and a particular sourcedependence encoded in $\hat{\alpha}_{\text {sat }}^{(\mathrm{mac})}$ we can then use eq. 5 . to determine the reduced microscopic saturation amplitudes $\hat{\alpha}_{\text {sat }}^{(\text {mic) }}$ from given pulsar timing data. The smallest value obtained from the entire data set, which is realized for a particular source with frequency $f_{0}$ and spindown rate $\dot{f}_{0}$, can then be used to give a limit for a general source, spinning with frequency $f$, using eqs. (4), (5) and (9). We find the universal r-mode spindown limit

ers of those parameters with no multiplicative prefactor. For the saturation mechanisms considered here there is a known set of macroscopic parameters (mass, radius, etc) but for generality we do not limit ourselves by writing $\alpha_{\mathrm{sat}}^{(\mathrm{mac})}$ explicitly in terms of them. $h_{0}^{(\text {usl })}=\sqrt{\frac{15}{4} \frac{G I\left|\dot{f}_{0}\right|}{D^{2} f_{0}}}\left(\frac{\hat{\alpha}_{\text {sat }}^{(\text {mac })}}{\hat{\alpha}_{\text {sat }, 0}^{\text {(mac) }}}\right)^{\frac{1}{1-2 \beta / \theta}}\left(\frac{f}{f_{0}}\right)^{\frac{3+\gamma+2 \beta / \theta}{1-2 \beta / \theta}}$.

The first factor is just the standard spindown limit eq. (10) for the source with the strongest bound on the reduced microscopic saturation amplitude, whereas the two others are correction factors involving information on the source to which this limit applies, with exponents $\beta, \gamma$, $\theta$ from eqs. (1) and (3). Note that this result is independent of the details of the cooling mechanism encoded in $\hat{L}$ although the effective spindown law eq. (5) that was used to obtain eq. 12 depends on it. It depends on the power law exponent $\theta$ of the cooling luminosity via the factor $2 \beta / \theta$, which takes different values depending on whether the cooling is dominated by neutrinos $(\theta=8$ for modified Urca cooling) or photons $(\theta=4)$. Recently it has been shown [13] that unless the dissipation is so strong that $r-$ modes are completely damped away, radio pulsars should be surprisingly hot due to the strong heating from r-mode dissipation. Therefore, both photon and neutrino cooling can be relevant for observed radio pulsars. Since the detailed properties of particular sources are generally unknown the ratio of the macroscopic parts of the reduced saturation amplitudes in eq. (12) can only be estimated. However, from our theoretical understanding of compact stars (possible range of masses, radii, etc) we can, for a given saturation mechanism, determine bounds on this unknown factor that are tight enough that the universal spindown limit is still considerably more restrictive than the standard spindown limit.

The simplest and most often used toy model for $\mathrm{r}$ mode saturation [11] assumes a constant saturation amplitude that is independent of both temperature and frequency, so $\beta=\gamma=0$. Although realistic models based on an explicit physical saturation mechanism have a more complicated dependence this simple case is useful for illustrative purposes. In this case the saturation amplitude is also assumed to be independent of the source so $\alpha_{\mathrm{sat}}=\hat{\alpha}_{\mathrm{sat}}^{\text {(mic) }}$, which is given in fig. 1. The strongest limit $\alpha_{\text {sat }} \leq 1.2 \times 10^{-7}$ is obtained for the fast pulsar J0034-0534 with $f_{0} \approx 533 \mathrm{~Hz}$ and $\dot{f}_{0} \approx-1.4 \times 10^{-15} \mathrm{~s}^{-1}$. Using this bound in eq. (8) shows that in the constant saturation model r-mode gravitational wave emission can only be present in sources spinning with frequencies $f \gtrsim 225 \mathrm{~Hz}$ corresponding to gravitational wave frequencies $\nu \gtrsim 300 \mathrm{~Hz}$, since slower spinning sources would have left the r-mode instability region (see fig. 2 and the accompanying discussion). The expression for the universal spindown limit shows that the bounds for other sources scale in this case as $\left(f / f_{0}\right)^{3}$. Therefore the universal spindown limits are significantly lower than the standard spindown limits since the saturation amplitude obtained from the entire data set is lower and the frequencies of most sources are lower than $f_{0}$. This is shown in fig. 5 which compares the universal spindown limits 


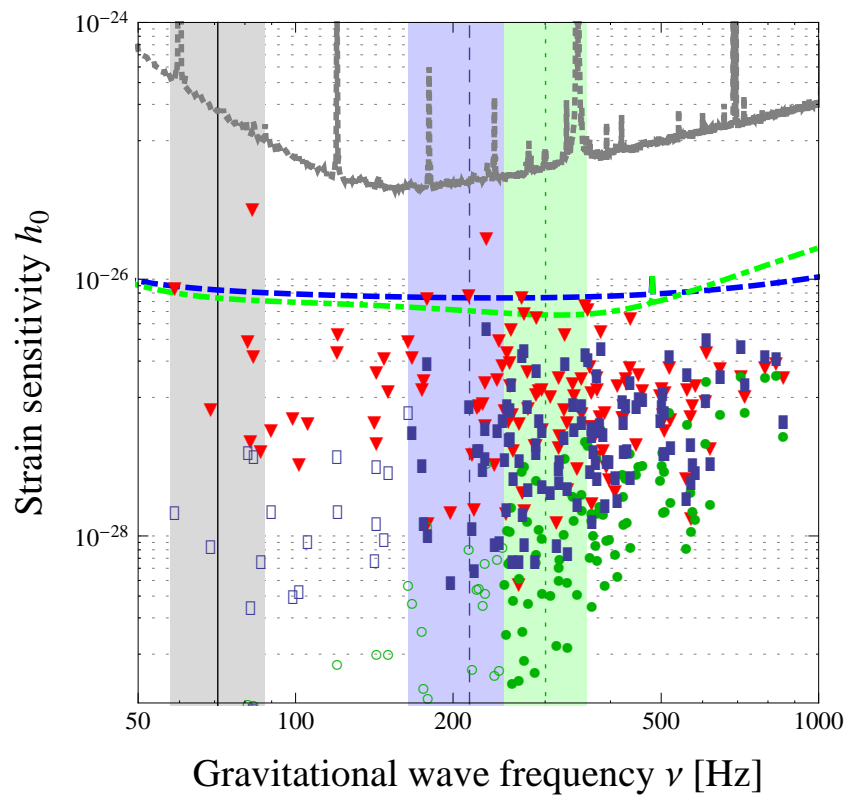

Figure 5: Comparison of different upper bounds on the strain amplitude of known radio pulsars due to r-mode emission. The spindown limit (red triangles) is obtained from the timing data of an individual source. The universal spindown limit takes into account that the saturation mechanism applies to the entire class of millisecond pulsars, and also provides a lower bound eq. (8) on the frequency. We show universal spindown limits (green circles) and minimum frequency (green dotted vertical line with uncertainty band) for the toy model of a constant r-mode saturation amplitude [1]. We also show universal spindown limits (blue rectangles) and minimum frequency (blue dashed vertical line with uncertainty band) for a realistic saturation mechanism arising from mode-coupling and the damping of the daughter modes by shear viscosity [17. For a given saturation mechanism, stars below the minimum frequency (open symbols) do not undergo r-mode oscillation.

for the constant saturation amplitude model (circles) to the standard spindown limits (triangles) given before in fig. 4. The, dashed vertical line in fig. 5 gives the universal $r$-mode frequency bound eq. 8 below which r-modes cannot be present. Therefore slower spinning sources, which appeared to be rather promising when only taking into account the standard spindown limit, are entirely excluded, as is denoted by the open symbols. But even for faster spinning sources the universal spindown limits can be orders of magnitude smaller. Therefore, all limits for this saturation mechanism are considerably below the estimated sensitivity of advanced LIGO.

R-mode saturation amplitudes obtained from realistic mechanisms have a temperature and/or frequency dependence. Moreover, the power law exponents that are found are generally negative and of order one. As an important realistic example we discuss the saturation due to mode coupling and the subsequent damping of the daughter modes [32]. The saturation amplitude from mode cou- pling has recently been revised [17, taking into account that the dominant damping source for daughter modes in a neutron star is likely shear viscosity instead of the previously assumed boundary layer damping. The revised saturation amplitude could be low enough to be compatible with the restrictive bounds from the observed small spindown rates given in fig. 1. In the case of a star with an impermeable crust the saturation amplitude is given by 17 .

$$
\alpha_{\mathrm{sat}}=\frac{\left|C_{R}\right|}{\sqrt{\tilde{J}}}=\left(1.4 \times 10^{-7} \frac{K_{4}^{\frac{2}{3}}}{\kappa_{D}}\right)\left(\frac{1}{\sqrt{\tilde{J}} R_{10}^{\frac{4}{3}}}\right) T_{8}^{-\frac{4}{3}} f_{500}^{-\frac{2}{3}} .
$$

Here the first parenthesis represents $\hat{\alpha}_{\text {sat }}^{\text {(mic) }}$, which can be determined from the spindown data. The lowest bound on $\hat{\alpha}_{\mathrm{sat}}^{\text {(mic) }}$ is obtained from the $f_{0}=336 \mathrm{~Hz}$ radio pulsar J2229+2643. Using this bound in eq. (8), in the mode coupling model $r$-modes are only present in sources that spin with frequencies $f \gtrsim 160 \mathrm{~Hz}$ corresponding to gravitational wave frequencies $\nu \gtrsim 215 \mathrm{~Hz}$. In the mode-coupling mechanism the saturation amplitude has a strong temperature and density dependence which gives a weaker scaling of the universal spindown limit. As seen in eq. 12 the scaling ranges from $\left(f / f_{0}\right)^{3 / 2}$ if neutrino cooling dominates to $f / f_{0}$ if photon cooling is dominant. To obtain rigorous upper bounds we are conservative and use for each source the weaker of the two constraints obtained from neutrino and photon cooling. The second parenthesis in eq. (13) is the source-dependent factor $\hat{\alpha}_{\mathrm{sat}}^{(\mathrm{mac})}$ which is unknown. To estimate the uncertainty in this factor, we note that the radius of a neutron star $R_{10}$ (in units of $10 \mathrm{~km}$ ) is at present uncertain within $1 \lesssim R_{10} \lesssim 1.5$ and the factor $\tilde{J}$, defined in [11, has been shown in [12] to be strictly bounded within $1 /(20 \pi) \leq \tilde{J} \leq 3 /(28 \pi)$. Therefore, the uncertainty on the universal spindown limit from the source-dependent factor in eq. 12 is $\hat{\alpha}_{\mathrm{sat}}^{(\mathrm{mac})} / \hat{\alpha}_{\mathrm{sat}, 0}^{(\mathrm{mac})} \lesssim 2.5$ Including this uncertainty, the results for the universal spindown limit for saturation due to mode-coupling are shown as well in fig. 5 (rectangles) and the corresponding frequency below which $\mathrm{r}$-modes are excluded is shown by the dashed vertical line. As can be seen the universal spindown limits are above those for the constant saturation model (circles), but in most cases still significantly below both the standard spindown limits and for all sources they are below the sensitivity of the advanced LIGO detector. For some fast spinning sources the standard spindown limit is more restrictive but these are far below the aLIGO sensitivity.

\section{CONCLUSIONS}

We have analyzed the continuous gravitational wave emission of millisecond radio pulsars due to r-modes. As an improvement to the usual bound, given by the spindown limit, we have derived the universal r-mode spin- 
down limit which takes into account the fact that proposed r-mode saturation mechanisms are insensitive to the macroscopic star configuration (mass, radius, moment of inertia, etc) and takes into account the whole class of sources. Using this additional information, we find that the universal spindown limit for the intrinsic gravitational wave strain amplitude can be significantly smaller than the usual spindown limit. Furthermore, we show that r-modes are damped in old millisecond radio pulsars spinning with frequencies below about $150-200 \mathrm{~Hz}$ so that corresponding gravitational wave emission is not expected to be present. Our results do not rely on explicit estimates for the r-mode saturation amplitude, which depend on the microphysics and are still very uncertain, but merely on the parametric temperature and frequency dependence which is generic for a given saturation mechanism and given by characteristic rational power-law exponents. We compare our improved bounds to the detection thresholds for realistic searches with next generation detectors like advanced LIGO using a year of coherent data and find that for none of the known millisecond radio pulsars would r-mode gravitational waves be detectable in the near future. This is in contrast to r-mode emission from young sources, where several potential sources are in reach of advanced LIGO [12]. However, if the sensitivity could be improved by the combination of different detectors, the analysis of larger coherent data sets or other enhancements, the universal spindown limits of selected millisecond pulsars which are close to the the detection limit might be beaten by next generation searches. For third generation detectors, like the planned Einstein telescope, there is a realistic chance to detect dozens of sources and our refined bounds identify those that are most promising. In contrast to the conventional spindown limit (triangles in fig. 5), which is sizable for some lower frequency sources, the universal spindown limit (circles or rectangles in fig. 5) shows that it is actually the mid- to high-frequency sources which feature the largest bounds.

The universal spindown limit relies on the assumption that the same r-mode saturation mechanism is operating in the entire set of radio pulsars. In principle it cannot be completely excluded that different saturation mechanisms are at work in different sources. This could happen if there were classes of sources with qualitatively different structural or phase compositions. For the recycled old radio pulsars that we focus on in this paper, this is not a likely scenario. These stars are very stable systems that hardly change over time and have very similar properties. For instance the magnetic fields that could dis- tinguish different radio pulsars are all rather small and are not expected to strongly affect the r-mode evolution besides the additional magnetic spindown. However, it is quite possible that old and young stars have different saturation mechanisms, in fact such a difference is required to explain the low spin frequencies of young pulsars [12] and the low spindown limits of old radio pulsars [13]. One possibility is enhanced dissipation due to the transformation of a neutron star or its core into a quark star owing to the density increase during the initial spindown [33. Another option would be enhanced dissipation in a superfluid/superconductor [19] which is only present below the superfluid melting temperature and this transition might have been explicitly observed in the cooling of the neutron star in Cassiopeia A 34. Both of these transitions would happen in the dynamic early evolution of young sources, before they are a few hundreds of years old. In contrast, recycled old radio pulsars are very stable systems that hardly change over time and have very similar properties. For instance the magnetic fields that could distinguish different radio pulsars are all rather small and are not expected to strongly affect the r-mode evolution besides the additional magnetic spindown. Therefore, the assumption that the same saturation mechanism is realized in the entire class of old millisecond radio pulsars is reasonable.

In addition to the exciting prospect of directly detectable gravitational waves, the emission from oscillating pulsars presents a unique chance to directly probe the interior of a compact star. The amplitude of r-modes, which is encoded in the gravitational wave signal, can directly reveal the damping properties of the matter inside the star and thereby its composition. In addition to thermal measurements from low mass x-ray binaries [10, 31. and pulsar timing data 6, 13, gravitational waves would provide a third messenger to probe the interior star composition via r-modes. The combined analysis of these different data sets would provide a clearer picture of the star's interior and could allow us to discriminate different star compositions in the future.

\section{Acknowledgments}

This research was supported in part by the Offices of Nuclear Physics and High Energy Physics of the U.S. Department of Energy under contracts \#DE-FG0291ER40628 and \#DE-FG02-05ER41375.
[1] LIGO Scientific Collaboration, G. M. Harry, Class.Quant.Grav. 27, 084006 (2010).

[2] LIGO Scientific Collaboration, Virgo Collaboration, A. J. Weinstein, Class.Quant.Grav. 29, 124012 (2012), 1112.1057 .
[3] LCGT Collaboration, K. Kuroda, Class.Quant.Grav. 27, 084004 (2010).

[4] LIGO Scientific Collaboration, Virgo Collaboration, J. Abadie et al., Phys.Rev. D85, 122007 (2012), 1202.2788 . 
[5] J. Aasi et al., (2013), 1309.4027.

[6] R. N. Manchester, G. B. Hobbs, A. Teoh, and M. Hobbs, Astron. J. 129, 1993 (2005), astro-ph/0412641.

[7] Virgo Collaboration, B. Abbott et al., Astrophys.J. 713, 671 (2010), 0909.3583.

[8] N. Andersson, Astrophys. J. 502, 708 (1998), grqc/9706075

[9] N. Andersson and K. D. Kokkotas, Int. J. Mod. Phys. D10, 381 (2001), gr-qc/0010102.

[10] L. Lindblom, B. J. Owen, and S. M. Morsink, Phys. Rev. Lett. 80, 4843 (1998), gr-qc/9803053

[11] B. J. Owen et al., Phys. Rev. D58, 084020 (1998), grqc/9804044

[12] M. G. Alford and K. Schwenzer, (2012), 1210.6091.

[13] M. G. Alford and K. Schwenzer, (2013), 1310.3524.

[14] LIGO Scientific Collaboration, J. Abadie et al., Astrophys.J. 722, 1504 (2010), 1006.2535.

[15] LIGO Scientific Collaboration, B. Abbott et al., Astrophys.J. 683, L45 (2008), 0805.4758.

[16] LIGO Collaboration, K. Wette et al., Class.Quant.Grav. 25, 235011 (2008), 0802.3332.

[17] R. Bondarescu and I. Wasserman, Astrophys.J. 778, 9 (2013), 1305.2335.

[18] M. G. Alford, S. Mahmoodifar, and K. Schwenzer, Phys.Rev. D85, 044051 (2012), 1103.3521.

[19] B. Haskell, K. Glampedakis, and N. Andersson, (2013), 1307.0985 .

[20] L. Lindblom, B. J. Owen, and G. Ushomirsky, Phys.Rev.
D62, 084030 (2000), astro-ph/0006242

[21] L.-M. Lin and W.-M. Suen, Mon. Not. Roy. Astron. Soc. 370, 1295 (2006), gr-qc/0409037

[22] L. Lindblom, J. E. Tohline, and M. Vallisneri, Phys. Rev. Lett. 86, 1152 (2001), astro-ph/0010653.

[23] Y. Wu, C. D. Matzner, and P. Arras, Astrophys.J. 549, 1011 (2001), astro-ph/0006123.

[24] L. Rezzolla, F. K. Lamb, and S. L. Shapiro, Astrophys.J. 531, L141 (2000), astro-ph/9911188

[25] S. Mahmoodifar and T. Strohmayer, (2013), 1302.1204.

[26] P. S. Shternin and D. G. Yakovlev, Phys. Rev. D78, 063006 (2008), 0808.2018.

[27] R. F. Sawyer, Phys. Rev. D39, 3804 (1989).

[28] M. Alford, S. Mahmoodifar, and K. Schwenzer, Phys.Rev. D85, 024007 (2012), 1012.4883.

[29] D. G. Yakovlev and C. J. Pethick, Ann. Rev. Astron. Astrophys. 42, 169 (2004), astro-ph/0402143.

[30] B. J. Owen, Phys.Rev. D82, 104002 (2010), 1006.1994.

[31] B. Haskell, N. Degenaar, and W. C. G. Ho, Mon. Not. Roy. Astron. Soc. 424, 93 (2012), 1201.2101.

[32] P. Arras et al., Astrophys. J. 591, 1129 (2003), astro$\mathrm{ph} / 0202345$.

[33] M. G. Alford and K. Schwenzer, PoS ConfinementX, 258 (2012), 1302.2649.

[34] D. Page, M. Prakash, J. M. Lattimer, and A. W. Steiner, Phys. Rev. Lett. 106, 081101 (2011), 1011.6142. 